\title{
Expression of additional transcription factors is of prognostic value for aggressive behavior of pituitary adenomas
}

\author{
Alexander Micko, MD, PhD, ${ }^{1}$ Thomas Rötzer, MD, ${ }^{2}$ Romana Hoftberger, MD, ${ }^{2}$ \\ Greisa Vila, MD, ${ }^{3}$ Johannes Oberndorfer, ${ }^{1}$ Josa M. Frischer, MD, PhD, ${ }^{1}$ Engelbert Knosp, MD, ${ }^{1}$ and \\ Stefan Wolfsberger, MD'
}

${ }^{1}$ Department of Neurosurgery, ${ }^{2}$ Institute of Neurology, and ${ }^{3}$ Department of Internal Medicine III, Clinical Division of Endocrinology and Metabolism, Medical University of Vienna, Austria

OBJECTIVE According to the latest WHO classification of tumors of endocrine organs in 2017, plurihormonal adenomas are subclassified by their transcription factor (TF) expression. In the group of plurihormonal adenomas with unusual immunohistochemical combinations (PAWUC), the authors identified a large fraction of adenomas expressing TFs for gonadotroph adenoma (TFGA) cells in addition to other TFs. The aim of this study was to compare clinicopathological parameters of PAWUC with TFGA expression to gonadotroph adenomas that only express TFGA.

METHODS This retrospective single-center series comprises 73 patients with TFGA-positive pituitary adenomas (SF1, GATA3, estrogen receptor $\alpha$ ): 22 PAWUC with TFGA (TFGA-plus group) and 51 with TFGA expression only (TFGA-only group). Patient characteristics, outcome parameters, rate of invasiveness (assessed by direct endoscopic inspection), and MIB1 and MGMT status were compared between groups.

RESULTS Patients in the TFGA-plus group were significantly younger than patients in the TFGA-only group (age 46 vs 56 years, respectively; $p=0.007$ ). In the TFGA-only group, pituitary adenomas were significantly larger (diameter 25 vs $18.3 \mathrm{~mm}, p=0.002$ ). Intraoperatively, signs of invasiveness were significantly more common in the TFGA-plus group than in the TFGA-only group ( $50 \%$ vs $16 \%, p=0.002$ ). Gross-total resection was significantly lower in the nonfunctioning TFGA-plus group than in the TFGA-only group ( $44 \%$ vs $86 \%, p=0.004)$. MIB1 and MGMT status showed no significant difference between groups.

CONCLUSIONS These data suggest a more aggressive behavior of TFGA-positive adenomas if an additional TF is expressed within the tumor cells. Shorter radiographic surveillance and earlier consideration for retreatment should be recommended in these adenoma types.

https://thejns.org/doi/abs/10.3171/2020.2.JNS2018

KEYWORDS plurihormonal adenoma; WHO classification; aggressive pituitary adenoma; transcription factor; pituitary surgery

$\mathrm{W}$ ITH the aim of differentiating pituitary adenomas according to their cell lineages, ${ }^{1,2}$ the latest WHO classification of tumors of endocrine organs in 2017 has included the transcription factors (TFs) PIT1 and TPIT and the TFs for gonadotroph adenoma (TFGA) cells SF1, GATA2/3, and estrogen receptor $\alpha$ in the classification of pituitary adenomas. In this context, plurihormonal adenomas are now defined by the immunohistochemical (IHC) expression of multiple hormones and further subclassified according to their TF expression pattern. Exceptions are mammosomatotroph (growth hormone [GH]-prolactin [PRL]) and gonadotroph adenomas ( $\beta$ follicle-stimulating hormone $[\beta-\mathrm{FSH}]-\beta$ luteinizing hormone $[\beta-\mathrm{LH}] \pm \alpha$ subunit).

According to the WHO 2017 classification, plurihormonal adenomas have been subclassified on the basis of their TF expression into two groups: 1) plurihormonal with one TF, termed "PIT1-positive adenomas" (previ-

ABBREVIATIONS ACTH = adrenocorticotropic hormone; ER = endocrine remission; FSH = follicle-stimulating hormone; GH = growth hormone; GKRS = Gamma Knife radiosurgery; GTR = gross-total resection; $\mathrm{IHC}=$ immunohistochemical; IQR = interquartile range; $\mathrm{LH}=$ luteinizing hormone; $\mathrm{MEN} 1$ = multiple endocrine neoplasia type 1; $\mathrm{oGTT}=$ oral glucose tolerance test; PAWUC = plurihormonal adenomas with unusual immunohistochemical combinations; $\mathrm{PRL}=$ prolactin; $\mathrm{SRL}=$ somatostatin receptor ligand; TF = transcription factor; TFGA = TF gonadotroph adenoma; TSH = thyroid-stimulating hormone.

SUBMITTED January 3, 2020. ACCEPTED February 10, 2020.

INCLUDE WHEN CITING Published online April 17, 2020; DOI: 10.3171/2020.2.JNS2018. 
ously known as silent subtype III pituitary adenoma); and 2) plurihormonal with more than one TF, termed "plurihormonal adenomas with unusual immunohistochemical combinations" (PAWUC).

Plurihormonal adenomas have been found to occur with higher frequency in younger patients when compared to other types of pituitary adenomas. In approximately $70 \%$ of cases, plurihormonal adenomas are discovered through their mass effect and do not show signs of hormonal activity. ${ }^{3}$ In the remaining cases, clinical effects most often reflect the presence of GH (acromegaly) and to a lesser extent adrenocorticotropic hormone (ACTH) overexpression (Cushing's disease) and PRL cells (hyperprolactinemia). A clinically relevant overexpression of glycoprotein hormone (thyroid-stimulating hormone [TSH], FSH, LH) production is rare. Furthermore, in patients with multiple endocrine neoplasia type 1 (MEN1), plurihormonal adenomas have a higher incidence rate than in non-MEN1 patients. ${ }^{4-6}$ Plurihormonal adenomas are more often macroadenomas than microadenomas, and signs of invasiveness have been reported in as many as $30 \%$ in PIT1-positive tumors. ${ }^{4}$ However, apart from PIT1-positive plurihormonal adenomas (IHC expression of GH, PRL, TSH $\pm \alpha$ subunit) that were identified to be more aggressive, little is known about the subgroup of PAWUC.

From our series of PAWUC, we identified a large fraction of adenomas expressing FSH/LH as well as the TFs for this cell lineage. Nonfunctioning adenomas in general, but also specifically the adenomas with immunocytochemical FSH/LH positivity, can exhibit very different biological behavior in terms of invasiveness and recurrence, ${ }^{6-8}$ and the expression of additional TFs to TFGA may be of prognostic value. The aim of this study was therefore to compare PAWUC with TFGA expression plus additional TFs to gonadotroph adenomas that only express the TFGA, in terms of biological behavior and clinical outcome.

\section{Methods}

Of all 458 pituitary adenomas surgically treated at our institution over 10 years (2008-2018), plurihormonal adenomas accounted for $5.7 \%$ (26 cases). This retrospective study comprises a single-center series of 73 patients with IHC-verified TFGA-positive pituitary adenomas: 22 of those with TFGA expression plus an additional TF (the TFGA-plus group), and 51 with TFGA expression only (the TFGA-only group). All patients were operated on using a purely endoscopic transsphenoidal approach.

Histopathological analysis was performed according to the criteria of the WHO 2017 classification of tumors of endocrine organs. ${ }^{2}$ The TFGA-plus group was defined as a combination of the TFGA TF (LH, FSH $\pm \alpha$ subunit) plus the PIT1 (GH, PRL, TSH) and/or TPIT (ACTH), corresponding to a subgroup of PAWUC. The TFGA-only group was defined as no other IHC TF expression additional to TFGA ( $\mathrm{LH}, \mathrm{FSH} \pm \alpha$ subunit) corresponding to gonadotroph adenomas.

Exclusion criteria were double adenomas (separate tumors with different cell types), combinations formerly classified as plurihormonal adenomas such as mammoso- matotroph adenomas (GH-PRL), solely PIT1-positive plurihormonal adenomas, or patients lost to follow-up. Furthermore, patients with TFGA only without showing an IHC expression of LH/FSH ( $\pm \alpha$ subunit) were excluded.

Hormone expression was classified as negative if $<1 \%$ of tumor cells stained positive by IHC. ${ }^{9}$ Hormone production was classified as active if hormonal levels were found higher than sex-adjusted upper limits and if stigmata of acromegaly, Cushing's disease, symptoms of hyperprolactinemia (which could not be explained by stalk effect), or secondary hyperthyroidism were present.

Histopathological examinations were performed (by T.R. and R.H.) without the knowledge of the extent of resection. Endocrine remission (ER) and gross-total resection (GTR) were assessed by endocrinologists and neurosurgeons (G.V., A.M., and S.W.) on the basis of follow-up hormone levels and follow-up MRI, blinded to the histopathological findings.

This study was approved by the Institutional Ethics Committee and was performed in accordance with the principles of the Declaration of Helsinki. The tumor samples were evaluated with the consent of the patients for further histopathological examination.

\section{Tissue Processing}

The surgical biopsy specimens were routinely processed and were immunohistochemically stained for pituitary hormones (ACTH, FSH, GH, LH, PRL, TSH, $\alpha$ subunit), TFs (PIT1, TPIT, SF1, GATA3, estrogen receptor $\alpha),{ }^{10,11}$ the proliferation marker MIB1, and the DNA repair protein MGMT. GATA3 was used for tissue processing instead of GATA2 due to the high degree of homologous amino acids of GATA2 and GATA3 and the widespread use of GATA3 antibodies in pathological analysis. ${ }^{12}$

All biopsy specimens were fixed in $4 \%$ neutral buffered formalin, routinely processed, embedded in paraffin, cut in 5- $\mu \mathrm{m}$ slices, and stained with $\mathrm{H} \& \mathrm{E}$. Immunohistochemistry was performed with a streptavidin-biotinperoxidase complex method. Two neuropathologists (T.R. and R.H.) separately reviewed specimens blinded to the intraoperative findings. Positive control tissues consisted of paraffin-embedded sections of appropriate positive controls for each antibody. Negative controls were assessed from nonrelevant antibodies of the same species and of the same immunoglobulin isotype (immunoglobulin G1). ${ }^{13}$

According to a study by Nishioka et al., ${ }^{9}$ TFs were considered positive only if a significant fraction of relevant tumor cells showed nuclear reactivity. In cases of plurihormonal adenomas, multiple TF expression was graded positive if a significant fraction of tumor cells showed a positive expression of gonadotroph tumor cells (SF1, GATA3, or estrogen receptor $\alpha$ ).

The MIB1 index, counted per 500 cells in the hotspot area, was used as a marker of the proliferation potential. MGMT expression status was assessed as the percentage of stained cells, i.e., $<10 \%$ (low), 10\%-50\% (moderate), and $>50 \%$ (high). ${ }^{13-16}$ Parasellar tumor extension was evaluated due to the revised Knosp classification. ${ }^{17}$ Tumor consistency was assessed based on the intraoperative status: soft tumors were defined as easy to remove with a suction device (easily suckable), whereas fibrous tumors showed 


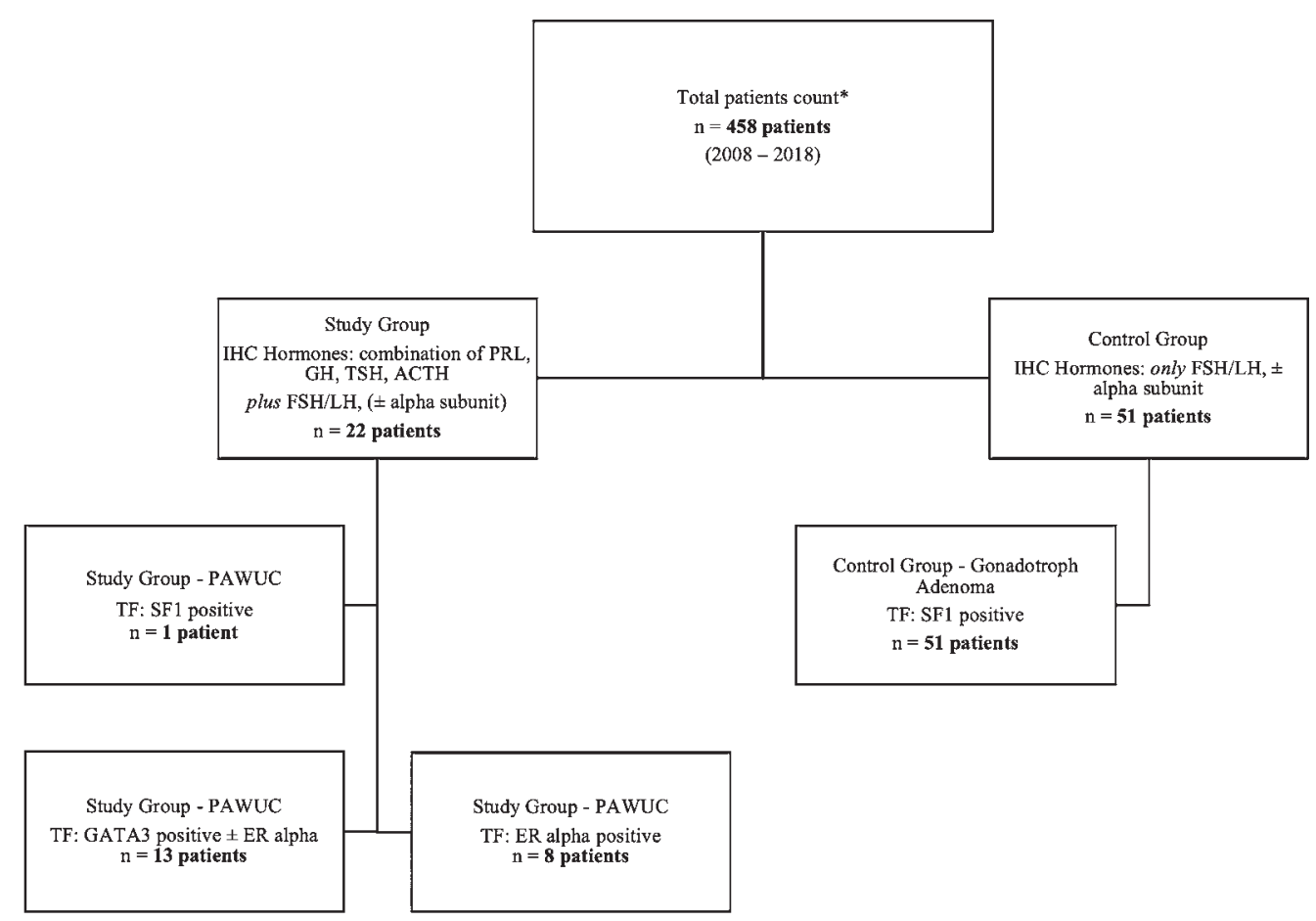

FIG. 1. Flowchart of patient characteristics. *Excluded: patients lost to follow-up, insufficient tumor tissue available for further IHC examinations.

a stiff and septated structure. ${ }^{18}$ The size (mean diameter) and tumor volume $\left(\mathrm{cm}^{3}\right)$ of the tumor were assessed on preoperative isovoxel planning MRI (StealthStation S8; Medtronic). As part of our routine clinical practice, invasiveness was judged intraoperatively by the neurosurgical team through direct endoscopic visualization. Basal sellar dura samples of the anterior sellar wall were additionally obtained for histological examination.

\section{Statistical Analysis}

The data are presented as mean (range) for continuous variables and as frequencies for categorical variables, as well as median (interquartile range [IQR]) for preoperative hormonal levels. To assess the difference in GTR/ER rate, the rate of invasiveness (additional basal sellar dura), MGMT status, and tumor consistency between groups, the chi-square test with Pearson's correlation coefficient was performed. To analyze a difference in Knosp high grade between groups, a Mann-Whitney U-test was applied. Differences in age at first surgery, tumor size (diameter, volume), and MIB1 index between the two groups were assessed by an unpaired Student t-test. A $p$ value $<0.05$ was considered significant. For statistical analyses, SPSS software (version 25.0; IBM Corp.) was used.

\section{Results}

\section{Study Cohort}

The study group of PAWUC with TFGA and additional TF expression (TFGA-plus group) consisted of 22 consecutive patients (22/458 cases, $4.8 \%)$. The control group (TFGA-only group) consisted of 51 patients with gonado- troph adenomas as confirmed by TFGA positivity on histopathology who were operated on and followed-up within the same time frame. Altogether, 73 consecutive patients were included in this study with a mean follow-up of 3.8 years (range $0.5-9$ years).

IHC TF analysis revealed the following subclass rates of TFGA-plus adenomas: 1) 20 TFGA/PIT1 patients $(\mathrm{FSH} / \mathrm{LH}$ plus $\mathrm{GH}, \mathrm{PRL} \mathrm{n}=9 ; \mathrm{FSH} / \mathrm{LH}$ plus $\mathrm{GH} \mathrm{n}=4$; $\mathrm{FSH} / \mathrm{LH}$ plus PRL $\mathrm{n}=3 ; \mathrm{FSH} / \mathrm{LH}$ plus PRL, TSH $\mathrm{n}=3$; FSH/LH, TSH $n=1) ; 2) 1$ TFGA/TPIT patient (FSH/LH plus $\mathrm{ACTH}$ ); and 3) 1 TFGA/PIT1/TPIT patient (FSH/ LH, GH, PRL, ACTH). For detailed information about TFGA distribution within groups, see Fig. 1.

\section{Comparison of Patient and Tumor Characteristics}

Patients in the TFGA-plus group were significantly younger than patients in the TFGA-only group (46 vs 56 years of age, $p=0.007$; Table 1). Sex distribution did not significantly differ within the groups (female/male ratio $=1: 1.2$ in the TFGA-plus group, 1:2.2 in the TFGA-only group; $\mathrm{p}=0.249$ ).

In the TFGA-only group, pituitary adenomas were significantly larger in diameter (25 vs $18.3 \mathrm{~mm}, \mathrm{p}=0.002$ ) at the time of diagnosis; however, tumor volume (9.8 vs $5.4 \mathrm{~cm}^{3}, \mathrm{p}=0.077$ ) only showed a trend to higher mean tumor volumes. However, this difference did not apply when comparing only nonfunctioning adenomas (TFGAonly vs TFGA-plus): diameter 25 vs $21.9 \mathrm{~mm}, \mathrm{p}=0.306$; volume 9.8 vs $7.0 \mathrm{~cm}^{3}, \mathrm{p}=0.457$.

In the TFGA-plus group, $13(59 \%)$ of 22 patients were found to harbor functioning active adenomas at the time of diagnosis: 11 cases of acromegaly (nadir oral glucose 
TABLE 1. Patient and tumor characteristics

\begin{tabular}{|c|c|c|c|}
\hline Characteristic & $\begin{array}{l}\text { PAWUC, } \\
\text { TFGA-Plus }\end{array}$ & TFGA-Only & $p$ Value \\
\hline No. of patients & 22 & 51 & \\
\hline 1st op & $19(86)$ & $47(92)$ & NS \\
\hline Recurrent op & $3(14)$ & $4(8)$ & \\
\hline Age (range), yrs & $46(20-72)$ & $56(27-75)$ & 0.007 \\
\hline$<40$ & $8(36)$ & $10(20)$ & NS \\
\hline$>40$ & $14(64)$ & $41(80)$ & \\
\hline Sex (F/M ratio) & $1: 1.2$ & $1: 2.2$ & NS \\
\hline Follow-up (range), yrs & $3.9(0.5-9)$ & $3.8(0.5-9)$ & NS \\
\hline \multicolumn{4}{|l|}{ Functional classification } \\
\hline Functional & $13(59)$ & - & $<0.001$ \\
\hline Nonfunctional & $9(41)$ & $51(100)$ & \\
\hline \multicolumn{4}{|l|}{ Tumor size (range) } \\
\hline Diameter, mm & $18.3(6-40)$ & $25.0(9-50)$ & 0.002 \\
\hline Volume, $\mathrm{cm}^{3}$ & $5.4(0.1-21.3)$ & $9.8(0.8-63.8)$ & 0.077 \\
\hline \multicolumn{4}{|l|}{ Tumor consistency } \\
\hline Soft & $19(86)$ & $47(92)$ & NS \\
\hline Fibrous & $3(14)$ & $4(8)$ & \\
\hline Invasiveness & $11(50)$ & $8(16)$ & 0.002 \\
\hline Knosp high grade & $10(45)$ & $22(43)$ & NS \\
\hline $3 \mathrm{~A}$ & $3(30)$ & $17(77)$ & NS \\
\hline 3B & $1(10)$ & $1(5)$ & \\
\hline 4 & $6(60)$ & $4(18)$ & \\
\hline Degree of resection & & & NS \\
\hline Complete & $17(77)$ & $47(92)$ & \\
\hline Subtotal, $>80 \%$ & $3(14)$ & $1(2)$ & \\
\hline Partial, $<80 \%$ & $2(9)$ & $3(6)$ & \\
\hline \multicolumn{4}{|l|}{ GTR } \\
\hline Functional & $7 / 13(54)$ & - & - \\
\hline Nonfunctional & $4 / 9(44)$ & $44(86)$ & 0.004 \\
\hline GKRS & $5(23)$ & $2(4)$ & 0.012 \\
\hline
\end{tabular}

NS = nonsignificant.

Data presented as mean (\%) unless otherwise indicated.

tolerance test [oGTT]: GH median $8.5 \mathrm{ng} / \mathrm{ml}, \mathrm{IQR} 6.3$ $22.2 \mathrm{ng} / \mathrm{ml}$; IGF-1 median $938 \mathrm{ng} / \mathrm{ml}$, IQR 542-1047 ng/ $\mathrm{ml}), 1$ case of acromegaly and hyperprolactinemia (nadir oGTT: GH 177 ng/ml, IGF-1 1493 ng/ml, PRL 464 ng/ml), and 1 case of hyperprolactinemia (PRL $350 \mathrm{ng} / \mathrm{ml}$ ). In the patient with only hyperprolactinemia, the indication for operation was resistance to dopamine agonist treatment and mass effect on the chiasm.

In the TFGA-only group $(\mathrm{n}=51)$, no patients exhibited any signs of hormone overexpression (functioning gonadotroph adenoma) and were operated on due to a mass effect of tumor. In recurrent cases, no tumor samples showed a different IHC hormonal or TF expression in comparison to the primary tumor.

When comparing parasellar extension on preoperative MRI between the groups, we more often found grade 3A in the TFGA-only group (77\% vs 30\%), and more often grade 4 in the TFGA-plus group (60\% vs 18\%). Although not significant, a clear trend for a different distribution of Knosp high-grade adenomas $(p=0.057)$ between groups could be found.

Intraoperatively, signs of invasiveness as determined by intraoperative endoscopic direct visualization were significantly more common in the TFGA-plus group than in the TFGA-only group (50\% vs $16 \%, \mathrm{p}=0.002)$. The histopathological examination of basal sellar dura invasiveness showed no statistical difference between the groups $(21 \%$ vs $22 \%, \mathrm{p}=0.951)$. There was no difference in fibrous tumor consistency between the groups $(14 \%$ in the TFGA-plus group vs $8 \%$ in the TFGA-only group, $p$ $=0.440$ ).

Overall, the GTR/ER rate was $75.3 \%$ (55/73); GTR/ER was significantly lower in the TFGA-plus group than in the TFGA-only group ( $50 \%$ vs $86 \%, p=0.001)$. GTR was significantly lower in the nonfunctional TFGA-plus group than in the TFGA-only group (44\% vs $86 \%, \mathrm{p}=0.004$ ).

Gamma Knife radiosurgery (GKRS) was performed in 2 of 5 patients in the TFGA-plus group and in 2 of 4 patients in the TFGA-only group with subtotal or partial tumor resection. The remaining 3 patients with subtotal or partial tumor resection in the TFGA-plus group had nonfunctioning adenomas and showed a stable tumor remnant after a second surgery. The same was found in 2 patients in the TFGA-only group.

In $8(11 \%)$ of the 73 patients, including $5(23 \%)$ of 22 in the TFGA-plus group and $3(6 \%)$ of 51 in the TFGA-only group in whom no remaining tumor tissue on early postoperative MRI and normal postoperative hormone levels were found, relapse occurred postoperatively at a median of 2.9 years (IQR $1.8-3.8$ years). In 5 (63\%) of 8 patients with relapse in the TFGA-plus group, 4 patients had a functioning tumor (acromegaly in all cases) and were further treated with long-acting somatostatin receptor ligands (SRLs), and in 3 (75\%) of 4 cases with GKRS. In 1 patient with nonfunctioning TFGA-plus adenoma and all patients in the TFGA-only group, a stable tumor remnant was noted during follow-up controls.

Evaluation of proliferation rate as assessed by the MIB1 index did not reveal any significant differences between the groups (3.8\% vs $2.9 \%$ for the TFGA-plus and TFGA-only groups, respectively; $p=0.164)$. Low MGMT immunoexpression $(<10 \%)$ was found in $7 \%(5 / 73)$ across all cases, including $80 \%(4 / 5)$ in the TFGA-plus group.

The frequency of low to moderate MGMT immunoexpression $(<50 \%)$ was $23 \%(17 / 73)$ across all cases. Low to moderate MGMT immunoexpression was not significantly differently distributed between groups (32\% in the TFGA-plus group vs $20 \%$ in the TFGA-only group, $\mathrm{p}=$ 0.257; Figs. 2 and 3).

\section{Discussion}

To the best of our knowledge, the current series represents the first description of TFGA-positive PAWUC after the WHO classification revision in 2017. Although of significantly smaller size than TFGA-only (gonadotroph) adenomas, we found a higher rate of invasiveness and of Knosp grade 4 parasellar growth, indicating a more ag- 


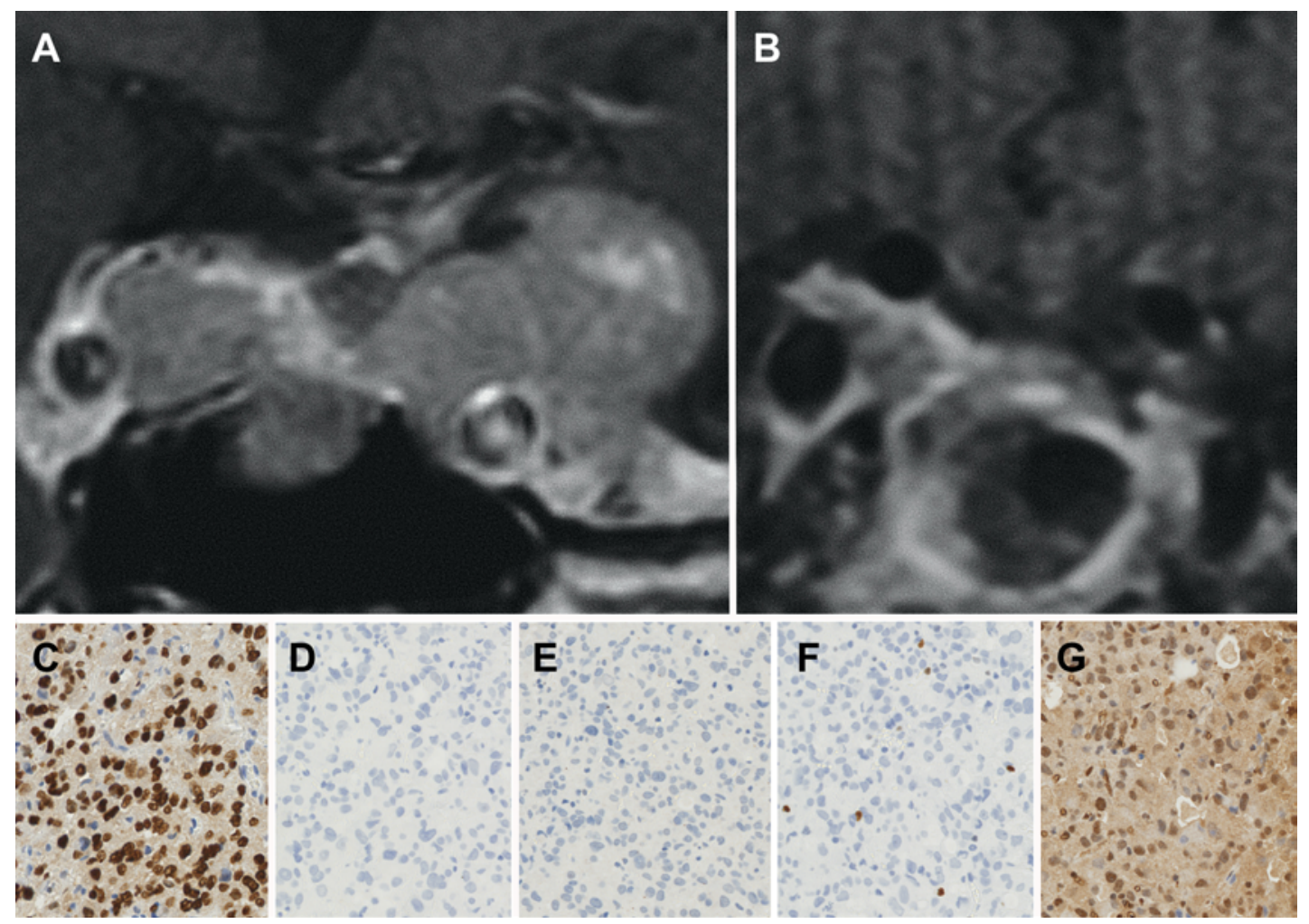

FIG. 2. Images of nonfunctioning PAWUC. A: Preoperative MR image depicting adenoma extension into the left cavernous sinus compartments. B: After a combination of 3 resections (2 transsphenoidal, 1 subfrontal) and 1 GKRS, 5-year postoperative MR image confirmed complete adenoma removal from the left cavernous sinus compartment. However, a recurrent tumor was noted on the right endo-/parasellar side that was treated by a second GKRS. C-G: IHC examination. PIT1 positive (C), TPIT negative (D), SF1 negative (E), MIB1 2.2\% (F), and MGMT > 50\% (G). Figure is available in color online only.

gressive behavior and, consequently, a lower rate of GTR/ ER in TFGA-positive plurihormonal adenomas.

\section{Plurihormonal Adenomas}

Prior to the widespread use of immunohistochemistry for detecting hormonal expression in pituitary adenomas, classification of pituitary adenomas was based on $\mathrm{H}$ \& $\mathrm{E}$ staining, which hampered the diagnosis of plurihormonal adenomas. ${ }^{19}$ With the revision of the 2004 WHO classification, immunohistochemistry has evolved to the gold standard in the diagnosis of pituitary adenomas as well as for defining pituitary adenoma subtypes. A possible benefit of using TFs to identify plurihormonal adenomas was presumed, but there were no sufficient available data for clarifying the cytological coherence. Furthermore, the group of subtype III adenomas was established (now termed "PIT1-positive adenomas").

Within the 2017 WHO classification, TFs have now been defined as a mainstay for diagnosing pituitary adenomas, especially in the case of plurihormonal adenomas. Due to these improvements in diagnosis, true "null cell adenomas," for example, are relatively rare. In an analysis by Nishioka et al., the authors accounted for only $0.6 \%$ of 1071 surgically treated adenomas. ${ }^{9}$ Many hormonenegative adenomas have been found to be TFGA-positive gonadotroph adenomas,${ }^{20}$ or in the case of plurihormonal adenomas, show an additional expression of TFGA (FSH, $\mathrm{LH} \pm \alpha$ subunit).

Several theories of how plurihormonal adenomas can possibly emerge have been published. On the one hand, the development may be based on a precursor cell that has undergone several transformation and/or mutation processes to advance to a plurihormonal adenoma. ${ }^{21-24}$ Alternatively, clone cells of an existing pituitary adenoma may undergo several multidirectional differentiations leading to the formation of this tumor entity. ${ }^{21,25,26}$ The difference of TF expression in plurihormonal adenomas has also been shown in our study cohort, as different types of TFGA (SF1, GATA3, estrogen receptor $\alpha$ ) have been expressed by the tumor cells (Fig. 1).

\section{PIT1-Positive Adenomas Versus PAWUC}

The prototype of monomorphous plurihormonal adenomas is PIT1-only-positive adenomas (formerly termed "silent subtype III adenoma"). Initially considered as a third variant of silent corticotroph adenoma, silent subtype III pituitary adenomas have been recognized as biologically aggressive tumors., ${ }^{3,27}$ PIT1-positive plurihormonal adenomas have been found with a frequency of $0.9 \%-3.2 \%$ in large series of resected cases. ${ }^{3,4}$ The series from the Mayo Clinic, St. Michael's Hospital, and the University Health Network showed that these neoplasms 

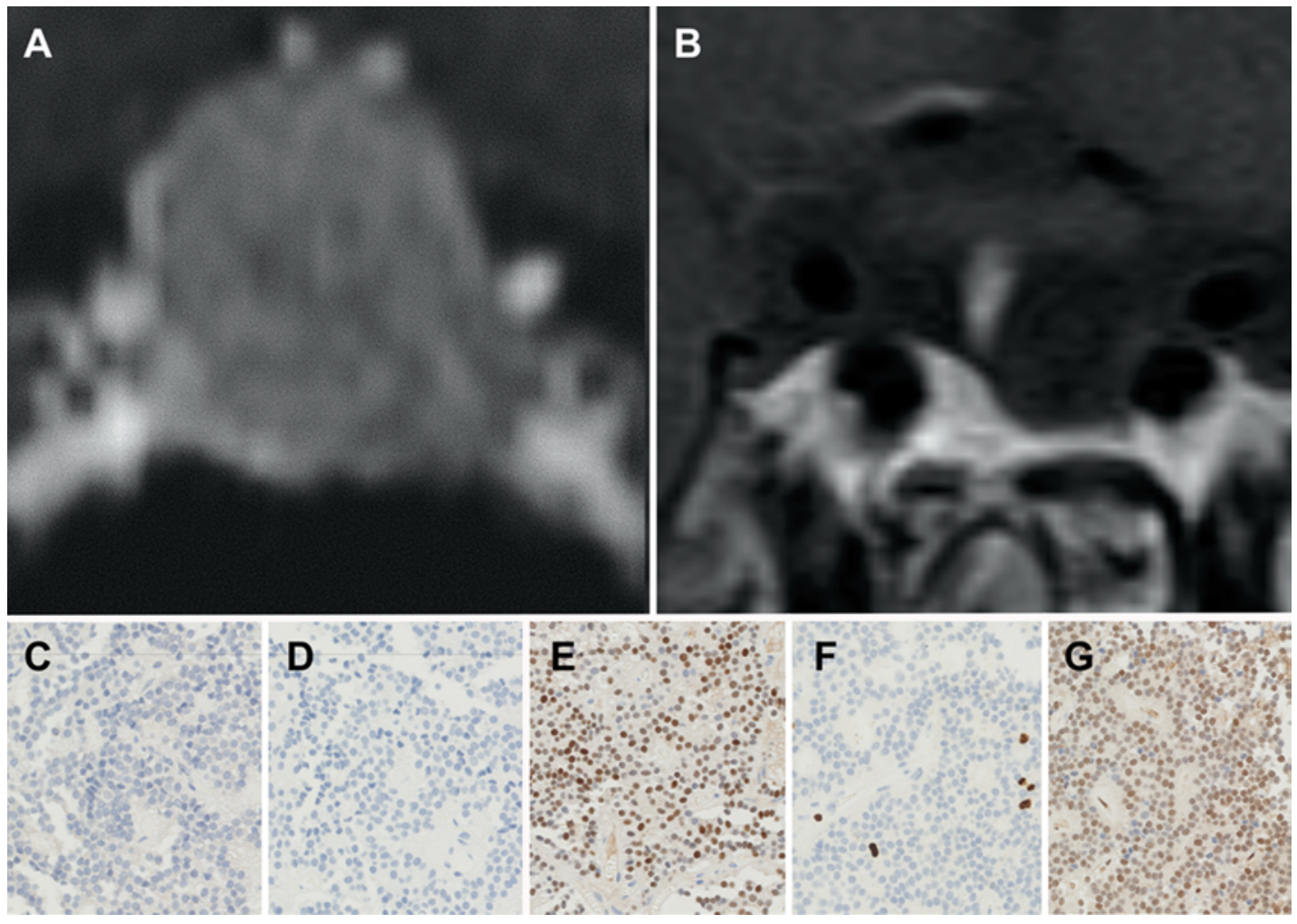

FIG. 3. Images of gonadotroph adenoma. A: Preoperative MR image depicting adenoma extension into the left cavernous sinus, Knosp grade 2. B: Postoperative MR image obtained 3 years after endoscopic transsphenoidal resection confirmed complete adenoma removal. C-G: IHC examination. PIT1 negative (C), TPIT negative (D), SF1 positive (E), MIB1 2.6\% (F), and MGMT $10 \%-50 \%(G)$. Original magnification $\times 40$. Figure is available in color online only.

were not always silent and may present with acromegaly, amenorrhea, and galactorrhea, as well as hyperthyroidism. Although a combination of clinical findings for different hormone oversecretions (e.g., acromegaly and hyperprolactinemia) is possible, it only occurs in rare cases.

The hallmark of these neoplasms is the expression of PIT1 and the presence of nuclear spheridia (nuclear inclusions). ${ }^{4}$ In contrast to differentiated PIT1 lineage adenomas (somatotroph, lactotroph, thyrotroph adenomas), these tumors often show a distinct IHC staining characterized by very focal or scattered positivity for one or more PIT1 hormones, including GH, PRL, and $\beta$-TSH.

In contrast to PIT1-only-positive plurihormonal adenomas, little is known about the subgroup of PAWUC. In a recently published retrospective cohort analysis, Aydin et al. reported PAWUC with a rate of $2.7 \%$ of all pituitary adenomas, ${ }^{28}$ in contrast to $4.8 \%$ in our series. The most common IHC combinations in PAWUC include GH, PRL, and one or more glycoprotein hormone subunits $(\beta-\mathrm{TSH}$, $\beta$-FSH, $\beta$-LH, and $\alpha$ subunit). ${ }^{29}$ In concordance with our findings, Aydin et al..$^{28}$ found the combination of PIT1 and TFGA to be the most common subtype.

In our series of 22 patients with PAWUC, 13 were hormone secreting. Of those, 12 (92\%) had acromegaly at the time of diagnosis, and 1 of these patients was diagnosed with additional hyperprolactemia $(464 \mathrm{ng} / \mathrm{ml})$. In this patient, the postoperative PRL concentration was within the normal range without dopamine agonist treatment.
However, this patient needed SRLs and GKRS postoperatively.

Due to the inclusion of TFs and improvements in IHC hormonal antibodies, many nonfunctioning plurihormonal adenomas that were formerly classified as null cell adenomas or silent adenomas of a single hormonal type have now been discovered to be plurihormonal adenomas. In our series, the most common subtype of TFGA-plus plurihormonal adenomas presented with a combination of $\mathrm{GH}$, PRL (PIT1), and FSH/LH (TFGA).

In 40\% (4/10) of nonfunctioning TFGA-plus PAWUC, a Knosp grade 4 parasellar invasion was found on preoperative MRI and therefore significantly more often than in the TFGA-only group (gonadotroph adenoma), with a frequency of $8 \%$. In cases of nonfunctioning PAWUC, considerations of shorter surveillance of tumor remnants and GKRS are recommended. Other combinations of TF hormones occur more rarely. Especially in cases of combinations with TPIT (such as ACTH), only small case series have been published. To date, 20 cases have been reported to co-express PIT1 and TPIT (GH and ACTH)..$^{30}$

It is common for GH- and ACTH-secreting pituitary tumors to show no clinical signs of Cushing's disease. In all reported cases of plurihormonal pituitary adenomas producing both $\mathrm{GH}$ and $\mathrm{ACTH}$, the dominant clinical characteristics have been those of GH overproduction. Only 3 cases of TFGA and TPIT (LH/FSH and ACTH) have currently been presented in the literature. In these adeno- 
mas, an increased risk of tumor recurrence was found to be associated with ACTH co-expression. ${ }^{31,32}$ Our series adds 2 more patients with TPIT and TFGA expression to the current literature (both nonfunctioning). One of these patients is currently in ER and the other showed relapse, with tumor regrowth.

\section{Markers for Plurihormonal Adenomas}

By analyzing the proliferation rate of plurihormonal adenomas, Pawlikowski et al. found significantly higher MIB1 values compared to monohormonal adenomas. ${ }^{33}$ However, in our series no significant difference in the MIB1 index was found between TFGA-plus plurihormonal (mean MIB1 index 3.8) and gonadotroph (mean MIB1 index 2.9) adenomas ( $\mathrm{p}=0.164)$.

In cases of PIT1-positive plurihormonal adenomas, MGMT IHC negativity was found in 50\% (5/10) of cases. ${ }^{34}$ In our 3 PIT1-positive plurihormonal adenoma cases, $33 \%(1 / 3)$ had a negative MGMT IHC expression. In cases of TFGA-plus plurihormonal adenomas, low MGMT immunoexpression $(<10 \%)$ was found in $80 \%(4 / 5)$ of cases in the TFGA-plus group. However, the frequency of low to moderate MGMT immunoexpression $(<50 \%)$ was not significantly differently distributed between groups $(32 \%$ in the TFGA-plus group vs $20 \%$ in the TFGA-only group, $\mathrm{p}=0.257$ ).

Richardson et al. found that plurihormonal adenomas had an upregulation of genes that encode for $A R G 2$ and $S E M A 3 A$, which regulate T-cell proliferation and function within the tumor microenvironment. This regulation of T-cell proliferation might be a possible target for future medical therapy in recurrent cases. ${ }^{35}$

\section{Limitations of the Study}

The main limitations of the presented study are due to the inherent nature of a retrospective analysis and the relatively small number of plurihormonal adenomas. Although a trend for a smaller tumor volume in the TFGAplus group was detected, no significant difference was found. However, tumor diameter is the common criteria for tumor size, which was found to be significantly different between the groups.

We were able to present the first comprehensive review of TFGA-positive plurihormonal adenomas with unusual hormonal combinations and report on the aggressive biological behavior of this subgroup of plurihormonal adenomas. Further multicenter studies are needed to investigate the entity of all subtypes of plurihormonal adenomas.

\section{Conclusions}

Our data suggest a more aggressive behavior of TFGApositive adenomas if an additional TF is expressed within the tumor cells, especially in cases of nonfunctioning PAWUC. This is particularly important as tumor size has failed to act as a marker to predict GTR/ER in tumors expressing TFGA. Detailed IHC testing of TFs and hormones is therefore important to make an accurate statement about the further behavior of pituitary adenomas. Consideration of shorter surveillance of tumor remnants and early radiosurgery are recommended in these adenoma subtypes.

\section{Acknowledgments}

We thank Gloria Wilk and Judith Ludwig for their assistance with immunohistochemical staining.

\section{References}

1. Kontogeorgos G, Kovacs K, Lloyd RV, Righi A. Plurihormonal and double adenoma. In: Lloyd RV, Osamura RY, Klöppel G, Rosai J, eds. WHO Classification of Tumours of Endocrine Organs. 4th ed. IARC; 2017:39-40.

2. Lloyd RV, Osamura RY, Klöppel G, Rosai J, eds. WHO Classification of Tumours of Endocrine Organs. 4th ed. IARC; 2017.

3. Erickson D, Scheithauer B, Atkinson J, et al. Silent subtype 3 pituitary adenoma: a clinicopathologic analysis of the Mayo Clinic experience. Clin Endocrinol (Oxf). 2009;71(1):92-99.

4. Mete O, Gomez-Hernandez K, Kucharczyk W, et al. Silent subtype 3 pituitary adenomas are not always silent and represent poorly differentiated monomorphous plurihormonal Pit-1 lineage adenomas. Mod Pathol. 2016;29(2):131-142.

5. Trouillas J, Labat-Moleur F, Sturm N, et al. Pituitary tumors and hyperplasia in multiple endocrine neoplasia type 1 syndrome (MEN1): a case-control study in a series of 77 patients versus 2509 non-MEN1 patients. Am J Surg Pathol. 2008;32(4):534-543.

6. Yamada S, Ohyama K, Taguchi M, et al. A study of the correlation between morphological findings and biological activities in clinically nonfunctioning pituitary adenomas. Neurosurgery. 2007:61(3):580-585.

7. Cooper O, Melmed S. Subclinical hyperfunctioning pituitary adenomas: the silent tumors. Best Pract Res Clin Endocrinol Metab. 2012;26(4):447-460.

8. Nishioka H, Inoshita N, Sano T, et al. Correlation between histological subtypes and MRI findings in clinically nonfunctioning pituitary adenomas. Endocr Pathol. 2012;23(3):151156.

9. Nishioka H, Inoshita $\mathrm{N}$, Mete $\mathrm{O}$, et al. The complementary role of transcription factors in the accurate diagnosis of clinically nonfunctioning pituitary adenomas. Endocr Pathol. 2015;26(4):349-355.

10. Lee JC, Pekmezci M, Lavezo JL, et al. Utility of Pit-1 immunostaining in distinguishing pituitary adenomas of primitive differentiation from null cell adenomas. Endocr Pathol. 2017;28(4):287-292.

11. Sjöstedt E, Bollerslev J, Mulder J, et al. A specific antibody to detect transcription factor T-Pit: a reliable marker of corticotroph cell differentiation and a tool to improve the classification of pituitary neuroendocrine tumours. Acta Neuropathol. 2017;134(4):675-677.

12. Mete O, Kefeli M, Çalışkan S, Asa SL. GATA3 immunoreactivity expands the transcription factor profile of pituitary neuroendocrine tumors. Mod Pathol. 2019;32(4):484-489.

13. Micko ASG, Wöhrer A, Höftberger R, et al. MGMT and MSH6 immunoexpression for functioning pituitary macroadenomas. Pituitary. 2017;20(6):643-653.

14. Kovacs K, Scheithauer BW, Lombardero M, et al. MGMT immunoexpression predicts responsiveness of pituitary tumors to temozolomide therapy. Acta Neuropathol. 2008;115(2):261-262.

15. Micko ASG, Höftberger R, Wöhrer A, et al. MGMT assessment in pituitary adenomas: comparison of different immunohistochemistry fixation chemicals. Pituitary. 2018;21(3):266-273.

16. Widhalm G, Wolfsberger S, Preusser M, et al. $\mathrm{O}^{6}$-methylguanine DNA methyltransferase immunoexpression in nonfunctioning pituitary adenomas: are progressive tumors potential candidates for temozolomide treatment? Cancer. 2009;115(5):1070-1080.

17. Micko AS, Wöhrer A, Wolfsberger S, Knosp E. Invasion of 
the cavernous sinus space in pituitary adenomas: endoscopic verification and its correlation with an MRI-based classification. J Neurosurg. 2015;122(4):803-811.

18. Micko A, Oberndorfer J, Weninger WJ, et al. Challenging Knosp high-grade pituitary adenomas [published online May 31, 2019]. J Neurosurg. doi:10.3171/2019.3.JNS19367

19. Kontogeorgos G. Classification and pathology of pituitary tumors. Endocrine. 2005;28(1):27-35.

20. Mete O, Asa SL. Clinicopathological correlations in pituitary adenomas. Brain Pathol. 2012;22(4):443-453.

21. Herman V, Fagin J, Gonsky R, et al. Clonal origin of pituitary adenomas. J Clin Endocrinol Metab. 1990;71(6):14271433.

22. Kovacs K, Horvath E, Stefaneanu L, et al. Pituitary adenoma producing growth hormone and adrenocorticotropin: a histological, immunocytochemical, electron microscopic, and in situ hybridization study. Case report. J Neurosurg. 1998;88(6):1111-1115.

23. Matsuno A, Sasaki T, Mochizuki T, et al. A case of pituitary somatotroph adenoma with concomitant secretion of growth hormone, prolactin, and adrenocorticotropic hormone-an adenoma derived from primordial stem cell, studied by immunohistochemistry, in situ hybridization, and cell culture. Acta Neurochir (Wien). 1996;138(8):1002-1007.

24. McComb DJ, Bayley TA, Horvath E, et al. Monomorphous plurihormonal adenoma of the human pituitary. A histologic, immunocytologic and ultrastructural study. Cancer. 1984;53(7):1538-1544.

25. Ho DM, Hsu CY, Ting LT, Chiang H. Plurihormonal pituitary adenomas: immunostaining of all pituitary hormones is mandatory for correct classification. Histopathology. 2001;39(3):310-319.

26. Kontogeorgos G, Scheithauer BW, Horvath E, et al. Double adenomas of the pituitary: a clinicopathological study of 11 tumors. Neurosurgery. 1992;31(5):840-849.

27. Horvath E, Kovacs K, Smyth HS, et al. Silent adenoma subtype 3 of the pituitary-immunohistochemical and ultrastructural classification: a review of 29 cases. Ultrastruct Pathol. 2005;29(6):511-524.

28. Aydin S, Comunoglu N, Ahmedov ML, et al. Clinicopathologic characteristics and surgical treatment of plurihormonal pituitary adenomas. World Neurosurg. 2019;130:e765-e774.

29. Rasul FT, Jaunmuktane Z, Khan AA, et al. Plurihormonal pituitary adenoma with concomitant adrenocorticotropic hormone $(\mathrm{ACTH})$ and growth hormone $(\mathrm{GH})$ secretion: a report of two cases and review of the literature. Acta Neurochir (Wien). 2014;156(1):141-146.

30. Roca E, Mattogno PP, Porcelli T, et al. Plurihormonal ACTHGH pituitary adenoma: case report and systematic literature review. World Neurosurg. 2018;114:e158-e164.
31. Bradley KJ, Wass JA, Turner HE. Non-functioning pituitary adenomas with positive immunoreactivity for ACTH behave more aggressively than ACTH immunonegative tumours but do not recur more frequently. Clin Endocrinol (Oxf). 2003;58(1):59-64.

32. Villa A, Cervasio M, Del Basso De Caro M, Cavallo LM. A rare case of ACTH-LH plurihormonal pituitary adenoma: letter to the editor. Acta Neurochir (Wien). 2014;156(7):13891391.

33. Pawlikowski M, Kunert-Radek J, Radek M. Plurihormonality of pituitary adenomas in light of immunohistochemical studies. Endokrynol Pol. 2010;61(1):63-66.

34. Salehi F, Scheithauer BW, Kros JM, et al. MGMT promoter methylation and immunoexpression in aggressive pituitary adenomas and carcinomas. J Neurooncol. 2011;104(3):647657.

35. Richardson TE, Shen ZJ, Kanchwala M, et al. Aggressive behavior in silent subtype III pituitary adenomas may depend on suppression of local immune response: a whole transcriptome analysis. J Neuropathol Exp Neurol. 2017;76(10):874882 .

\section{Disclosures}

Dr. Wolfsberger is currently an educational consultant for Medtronic Surgical Technologies.

\section{Author Contributions}

Conception and design: Wolfsberger, Micko. Acquisition of data: Micko, Rötzer, Vila, Oberndorfer. Analysis and interpretation of data: Micko, Rötzer, Vila. Drafting the article: Micko. Critically revising the article: Wolfsberger, Micko, Rötzer, Hoftberger, Vila, Frischer, Knosp. Reviewed submitted version of manuscript: all authors. Approved the final version of the manuscript on behalf of all authors: Wolfsberger. Statistical analysis: Micko. Administrative/technical/material support: Rötzer, Oberndorfer. Study supervision: Wolfsberger.

\section{Supplemental Information Online-Only Content}

Supplemental material is available with the online version of the article.

Supplemental Table 1. https://thejns.org/doi/suppl/10.3171/ 2020.2.JNS2018.

\section{Correspondence}

Stefan Wolfsberger: Medical University of Vienna, Austria. stefan. wolfsberger@meduniwien.ac.at. 